\title{
Mesenchymal Chondrosarcoma of maxilla: A rare case report
}

\author{
Vipul Jaetli ${ }^{1}$, Sunita Gupta ${ }^{2}$
}

${ }^{1}$ MDS Senior Resident, Department of Oral Medicine, Diagnosis and Radiology, Maulana Azad Institute of Dental Sciences, New Delhi, India

${ }^{2}$ MDS Professor and Head; Department of Oral Medicine, Diagnosis and Radiology, Maulana Azad Institute of Dental Sciences, New Delhi, India

Correspondence: B-3/6 Rana Pratap Bagh, New Delhi- 110007 India, jetlyvipul@gmail.com

Received: 01/03/2010 Accepted: $31 / 05 / 2010$

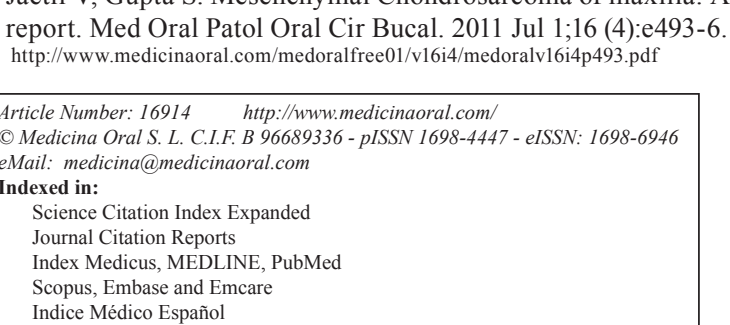
report. Med Oral Patol Oral Cir Bucal. 2011 Jul 1;16 (4):e493-6. http://www.medicinaoral.com/medoralfree01/v16i4/medoralv16i4p493.pdf

\begin{abstract}
Mesenchymal chondrosarcoma (MC) is a rare variant of chondrosarcoma (CS) that accounts for upto 3-9\% of all $\mathrm{CS}$ and has high predilection for the head and neck region. It is usually seen in younger age group compared to conventional CS and maxillary anterior alveolus is the most common site. The tumor is most unusual as it has been described as a particularly aggressive neoplasm with a high tendency for late recurrence and delayed metastasis. It is a biphasic tumor with areas comprising of spindle cell mesenchyme interspread with areas of chondroid differentiation. A 75 year old male presented to us as a painless mass in maxilla. Contrast enhanced computed tomography (CECT) revealed a lytic expansile lesion in the left maxillary bone with foci of calcification within soft tissue lesion. Fine needle aspiration cytology (FNAC) and incisional biopsy was performed which confirmed the diagnosis of maxillary MC. The patient underwent right and left subtotal maxillectomy with $2 \mathrm{~cm}$ margins. The review of literature shows that very few cases of maxillary MC have been reported so far. Thus an attempt is made to add this rare case of MC of maxillary alveolus in the English literature.
\end{abstract}

Key words: Chondrosarcoma, chondroid maxilla, mesenchymal chondrosarcoma, spindle cell.

\section{Introduction}

Chondrosarcoma (CS) is a malignant tumor characterized by the formation of cartilage and usually arises in peripheral long and flat bone $(1,2)$. It accounts for approximately

$10-20 \%$ of malignant bone tumors (2). Head and neck $\mathrm{CS}$ is an uncommon entity accounting for approximately $0.1 \%$ of all head and neck cancers (3). Several variants of CS have been proposed including clear cell, dedifferentiated, myxoid and mesenchymal (3-5). Mesenchymal chondrosarcoma (MC) is a rare variant of
CS that accounts for up to $3-9 \%$ of all CS and has high predilection for the head and neck region. It is usually seen in younger age group compared to conventional CS (6). The tumor is most unusual as it has been described as a particularly aggressive neoplasm with a high tendency for late recurrence and delayed metastasis $(3,6)$. The review of literature shows that a very few cases of maxillary MC have been reported so far in the English literature. Here an additional rare case of $\mathrm{MC}$ of maxillary alveolus in 75 year old patient is reported and discussed. 


\section{Case Report}

A 75 year old male patient presented to our department with a chief complaint of a painless growth in the left maxillary alveolar region since 4 months. The patient was apparently well 4 months back when he noticed a small growth in his left maxillary alveolus which grew rapidly to the present dimensions. As the lesion was asymptomatic initially the patient neglected it but as it continued to grow in size he became concerned and visited our institute for the management of the same. His medical history revealed that he was hypertensive since 6 years and was on regular medications for the same. Also, he had been operated for inguinal hernia 2 years back. Extraoral examination revealed a well defined firm swelling in relation to upper anterior face raising the upper lip. It extended from ala of the nose superiorly to vermillion of upper lip inferiorly and midline of nose anteriorly to a line drawn downwards from the midpupillary point posteriorly on the left side of face. The skin over the swelling was smooth and appeared to be stretched with no surface ulcerations or raised temperature. Intraoral examination revealed two separate lobulated swellings arising from the left maxillary alveolar region (Fig. 1). The buccal swelling extended anterior-posteriorly from distal aspect of 21 to mesial aspect of 26 and medio-laterally from buccal sulcus to palatal alveolus causing expansion of the buccal cortical plate and obliterating the buccal sulcus. The swelling measured approximately about $2 \times 3 \mathrm{~cm}$ in dimensions and caused gross buccal displacement of 23 and 24 . The palatal swelling extended anterior-posteriorly from mesial aspect of 21 to distal aspect of 26 and medially from $0.5 \mathrm{~cm}$ from mid-palatal region on the same side to the palatal alveolus laterally measuring approximately $1.5 \times 3 \mathrm{~cm}$ in dimensions. The buccal swelling was firm to hard in consistency with mucosa over swelling smooth and stretched. The palatal swelling was firm in consistency with superficial surface necrosis. There was no significant associated lymphadenopathy associated with the lesion. FNAC from the lesion was carried out which revealed chondroid matrix suggesting chondroid tumor. CECT revealed a lytic expansile lesion in the left maxillary bone extending medially upto midline and laterally extending into gingivobuccal sulcus (Fig. 2). Anterioposterior extension was from central incisor to first molar on the left side. There were foci of calcification within soft tissue lesion but there was no evidence of significant lymphadenopathy.

To confirm the diagnosis incisional biopsy was undertaken. While giving incision on the lesion a hard calcified tissue was felt which was included in the biopsy along with the soft tissue specimen. Histopathology study revealed cellular connective tissue stroma with spindle shaped cells arranged in lobular pattern. The cells showed dysplastic features with increased mitotic figures. Large areas of chondroid matrix with areas of calcification were also seen (Fig. 3). Overall histopathologic picture suggested mesenchymal chondrosarcoma of maxilla.

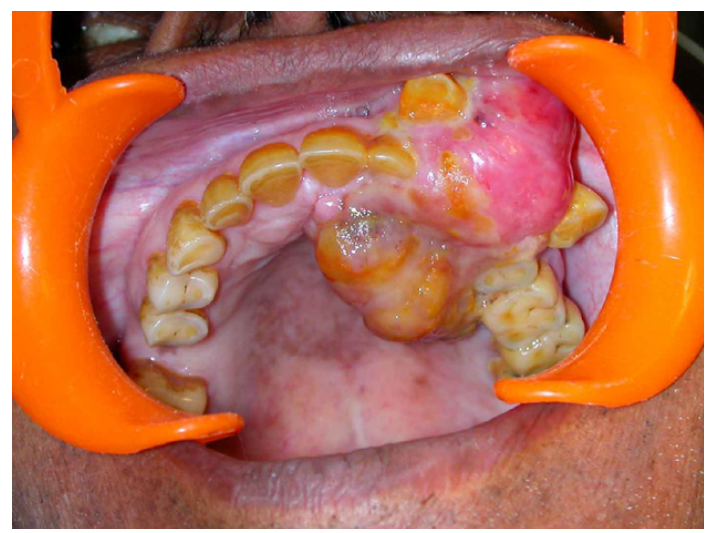

Fig. 1. Intraoral view of the lesion showing bucco-palatal extension and causing displacement of 23 and 24 buccally.

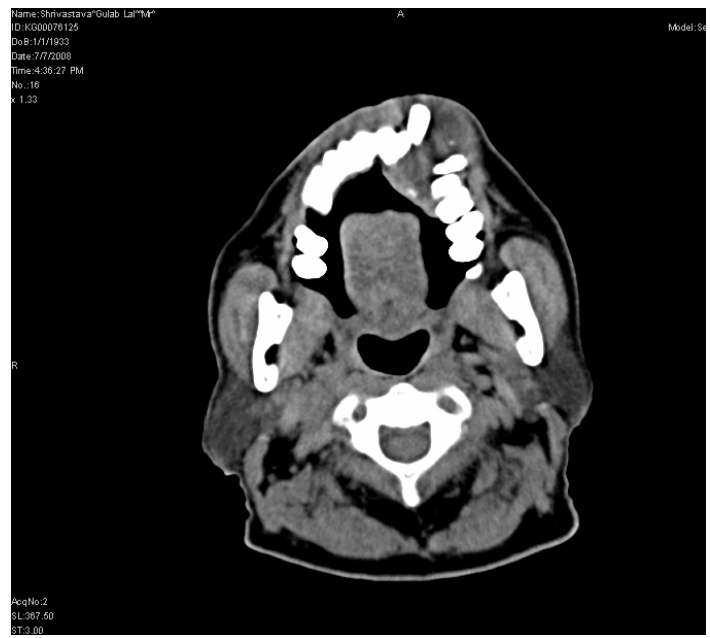

Fig. 2. CT scan (axial view) shows bucco-palatal extension of the lesion with displacement of 23 and 24 and foci of calcifications both buccally and palatally.

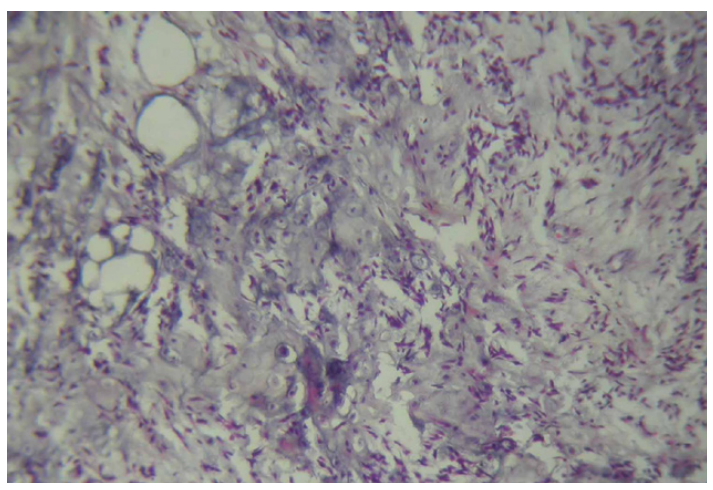

Fig. 3. Histopathology revealed cellular connective tissue stroma with spindle shaped cells interspersed with lacunar cells in a chondroid matrix with areas of calcification. 
The patient was operated under general anesthesia and underwent right and left subtotal maxillectomy taking $2 \mathrm{~cm}$ margin overall and remnants of nasal mucosa was sutured with antral mucosa. The palatal defect was closed with a medicated gauze pack place under palatal surgical splint. Post healing palatal obturator was fabricated to close oro-nasal communication. The patient is under observation and after 9 months of follow up is doing well.

\section{Discussion}

$\mathrm{MC}$ is a rare variant of CS that was first described by Lichenstein and Bernstein in 1959 as a biphasic tumor with areas comprising of spindle cell mesenchyme interspread with areas of chondroid differentiation (3, 5-8). Although considered to be rare the jaw bones constitute the most common location for MC $(7,9)$.

CS has been considered to be malignant tumor histogenetically derived from mature cartilaginous tissue (10). Since maxilla is a bone of exclusive membranous ossification; the possibility of CS is therefore surprising and has been explained by the vestigial nests of cartilage having source from (11)

1. cartilage found in incisive papilla

2. vestigial cartilage remnants in periodontal ligament

3. foci of cartilage from cartilaginous nasal capsule

4. paraseptal cartilage.

The present school of thought, however, says that CS may be derived either from cartilaginous tissue reserve cells (primary CS) or from primitive mesenchymal cells displaying chondromatous differentiation. Thus it explains that CS maxilla is derived from cartilaginous differentiation of primitive mesenchymal cells rather than from embryonal cartilaginous nests (10).

Most chondrosarcomas of the head and neck region occur in the maxilla; others are found in descending order of frequency in the body of the mandible, the ramus, the nasal septum, and the paranasal sinuses. In the maxilla the most common location is anterior alveolus where pre-esisting nasal cartilage is present $(12,13)$. Our reported case initially occurred in maxillary anterior region but as the lesion expanded it involved posterior part of jaw as well.

Although CS occurs most often in $3^{\text {rd }}-6^{\text {th }}$ decade of life, the $\mathrm{MC}$ variant occurs in $2^{\text {nd }}-3^{\text {rd }}$ decade of life in $70 \%$ of cases $(6,9,12)$. Very few cases have been reported to occur beyond the $6^{\text {th }}$ decade $(5,6)$. Our reported case falls in this rare category of occurrence beyond the $6^{\text {th }}$ decade of life.

There is some discrepancy in the literature regarding gender distribution in cases of MC. There are some reports indicating equal prevalence between sexes while others showing a slight male prevalence (5). Our reported case occurred in a male patient.

The most common clinical presentation of MC of jaws is a painless mass or swelling in comparison to its occurrence elsewhere in the body where pain and swelling are the most common presenting symptoms $(6,12-15)$. In a study of $36 \mathrm{MC}$ of maxilla by Niven et al. the most common reported symptom was swelling/mass in $68 \%$ of cases, followed by nasal obstruction (32\%), epistaxis (32\%) and tooth mobility ( $24 \%$ ) (7). Our reported case also presented to us as a painless mass in the palate with grade I mobility of the involved teeth.

In the same study by Niven et al. they found that the duration of signs and symptoms before diagnosis ranged from 2 weeks- 4 years and in most cases, did not exceed but a few months. Our case was presented and diagnosed after a period of 4 months (7).

The radiographic features of $\mathrm{MC}$ are not characteristic. The tumor usually shows features of a malignancy, consisting of a radiolucent process with poorly defined borders. The radiolucent area often contains scattered foci, which are caused by calcification or ossification of the cartilage matrix. It often demonstrates extensive infiltration between the osseous trabeculae of the preexisting bone without causing appreciable resorption. The lesion may also cause symmetrical widenening of the periodontal ligament space of the involved teeth (14). Our case in discussion showed similar radiographic appearance of an ill defined lytic lesion with radio-opaque sclerotic foci in the maxillary alveolus without causing root resorption.

The histologic appearance of MC is characteristically biphasic pattern. Undifferentiated areas appear as sheets of primitive mesenchymal spindle/round cells similar to small cell anaplastic sarcoma. However, islands of relatively well differentiated cartilaginous tumor help in making a specific diagnosis (6). Calcification or ossification may occur within the chondroid matrix. Neoplastic cartilage may be replaced by bone in a manner similar to normal endochondral ossification. Histologically the lesion must be differentiated from similar other lesions like hemangiopericytoma, Ewing's sarcoma, PNET, leukemia/lymphoma, rhabdomyosarcoma, malignant melanoma and small cell osteocarcoma (7). Evans and coworkers have classified CS into grades I, II and III on the basis of mitotic rate, cellularity and nuclear size (12). Grade I lesions resembles benign cartilage and they do not metastasize. Grade II lesions demonstrate more myxoid stroma. They recur locally more often than grade I lesions and have $10 \%$ incidence of metastasis. Grade III lesions have a more cellular pleomorphic appearance. They have a spindle cell proliferation with a marked increase in number of mitotic figures. The incidence of metastasis in these lesions is more than $70 \%$ (1). Our case showed histologic picture of highly cellular CT stroma of spindle/stellate cells with increased mitotic figures suggestive of grade III lesion.

With regards to therapy, various treatment modalities 
have been reviewed. The treatment of choice is surgical resection with wide margins. The overall low incidence of regional metastasis may suggest that neck dissection is not indicated $(13,14)$. Radiotherapy might play a role when accompanied by surgery although some believe $\mathrm{MC}$ to be a radioresistant tumor. Chemotherapy has a limited role in chondrosarcoma and should be used as an adjuvant therapy in cases with aggressive behavior with potential metastasis, rapid local recurrence and high grade lesions $(5,13)$.

In general the prognosis for patients with $\mathrm{MC}$ is poor because of the tumor's tendency to local recurrences or metastasis even after a long disease free interval. An interval as a long as 20 years have been reported before recurrence or metastasis occurred. The principal sites of metastasis are the lungs and bone. The reported 5 year and 10 year survival rate is $48 \%$ and $28 \%$ respectively (5).

Although the patient in present case was treated surgically with wide margins of resection and is doing well presently but knowing the notorious nature of $\mathrm{MC}$ in jaws the patient is kept under a close long term follow up.

\section{References}

References with links to Crossref - DOI

1. Selz PA, Konrad HR, Woolbright E. Chondrosarcoma of the maxilla: a case report and review. Otolaryngol Head Neck Surg. 1997;116:399-400.

2. Munshi A, Atri SK, Pandey KC, Sharma MC. Dedifferentiated chondrosarcoma of the maxilla. J Cancer Res Ther. 2007;3:53-5.

3. Knott PD, Gannon FH, Thompson LD. Mesenchymal chondrosarcoma of the sinonasal tract: a clinicopathological study of 13 cases with a review of the literature. Laryngoscope. 2003;113:783-90.

4. Garrington GE, Collett WK. Chondrosarcoma. I. A selected literature review. J Oral Pathol. 1988;17:1-11.

5. Angiero F, Vinci R, Sidoni A, Stefani M. Mesenchymal chondrosarcoma of the left coronoid process: report of a unique case with clinical, histopathologic, and immunohistochemical findings, and a review of the literature. Quintessence Int. 2007;38:349-55.

6. Zakkak TB, Flynn TR, Boguslaw B, Adamo AK. Mesenchymal chondrosarcoma of the mandible: case report and review of the literature. J Oral Maxillofac Surg. 1998 ;56:84-91.

7. Tien N, Chaisuparat R, Fernandes R, Sarlani E, Papadimitriou JC, Ord RA, et al. Mesenchymal chondrosarcoma of the maxilla: case report and literature review. J Oral Maxillofac Surg. 2007;65:1260-6.

8. Aziz SR, Miremadi AR, McCabe JC. Mesenchymal chondrosarcoma of the maxilla with diffuse metastasis: case report and literature review. J Oral Maxillofac Surg. 2002;60:931-5.

9. Vencio EF, Reeve CM, Unni KK, Nascimento AG. Mesenchymal chondrosarcoma of the jaw bones: clinicopathologic study of 19 cases. Cancer. 1998;82:2350-5.

10. Massarelli G, Gandolfo L, Tanda F, Ghiselli F, Manunta V. Maxillary chondrosarcoma (report of two cases). J Laryngol Otol. 1988;102:177-81.

11. Paterson W. Chondrosarcoma of the maxilla. J Laryngol Otol. 1955;69:132-9.

12. Hackney FL, Aragon SB, Aufdemorte TB, Holt GR, Van Sickels JE. Chondrosarcoma of the jaws: clinical findings, histopathology, and treatment. Oral Surg Oral Med Oral Pathol. 1991;71:139-43.

13. Gallego L, Junquera L, Fresno MF, De Vicente JC. Chondrosarcoma of the temporomandibular joint. A case report and review of the literature. Med Oral Patol Oral Cir Bucal. 2009;14:E39-43.

14. Garrington GE, Collett WK. Chondrosarcoma. II. Chondrosarcoma of the jaws: analysis of 37 cases. J Oral Pathol. 1988;17:12-20.
15. Mateos M, Forteza G, Gay-Escoda C. Mesenchymal chondrosarcoma of the maxilla. A case report. Int J Oral Maxillofac Surg. 1997;26:210-1 